\title{
Mechanical behavior of osteoporotic bone at sub-lamellar length scales
}

\author{
Ines Jimenez-Palomar ${ }^{1}$, Anna Shipov ${ }^{2}$, Ron Shahar ${ }^{2}$ and Asa H. Barber ${ }^{1,3}$ * \\ ' School of Engineering and Materials Science, Queen Mary University of London, London, UK \\ ${ }^{2}$ Koret School of Veterinary Medicine, The Hebrew University of Jerusalem, Jerusalem, Israel \\ ${ }^{3}$ School of Engineering, University of Portsmouth, Portsmouth, UK
}

Edited by:

Federico Bosia, University of Torino, Italy

\section{Reviewed by:}

Guy M. Genin, Washington University in St. Louis, USA

Fei Hang, South China University of Technology, China

\section{${ }^{*}$ Correspondence:}

Asa H. Barber, School of Engineering, University of Portsmouth, Portsmouth PO1 2UP, UK

e-mail: asa.barber@port.ac.uk
Osteoporosis is a disease known to promote bone fragility but the effect on the mechanical properties of bone material, which is independent of geometric effects, is particularly unclear. To address this problem, micro-beams of osteoporotic bone were prepared using focused ion beam microscopy and mechanically tested in compression using an atomic force microscope while observing them using in situ electron microscopy. This experimental approach was shown to be effective for measuring the subtle changes in the mechanical properties of bone material required to evaluate the effects of osteoporosis. Osteoporotic bone material was found to have lower elastic modulus and increased strain to failure when compared to healthy bone material, while the strength of osteoporotic and healthy bone was similar. Surprisingly, the increased strain to failure for osteoporotic bone material provided enhanced toughness relative to the control samples, suggesting that lowering of bone fragility due to osteoporosis is not defined by material performance. A mechanism is suggested based on these results and previous literature that indicates degradation of the organic material in osteoporosis bone is responsible for resultant mechanical properties.

Keywords: bone, osteoporosis, micromechanics, AFM, FIB

\section{INTRODUCTION}

Osteoporosis is one of the most significant types of bone disease that causes degradation of bone's mechanical function. Osteoporosis is characterized by significant changes in bone structure causing increases in bone fragility and therefore an increase in fracture risk (Kilbanski et al., 2001). The clinical importance of osteoporosis has been vigorously investigated in recent years due to the amount of people affected. In the United States alone, the costs of fractures resulting from osteoporosis have been estimated to be from 10 to 18 billion dollars per year and are expected to increase to 60 billion by the year 2020 (Iacono, 2007). The effects of osteoporosis on bone are characterized by two distinct forms; Type I, which refers to the loss of trabecular bone mass after menopause due to lack of estrogen and Type II, which refers to loss of cortical and trabecular bone in both men and women as a result of aging (Marcus and Bouxsein, 2010). The disturbances in osteoporotic bone structure are known to be due to changes in metabolic conditions such as hormonal changes (decrease in estrogen levels, growth hormone deficiency, increase in parathyroid hormone), steroids (glucocorticoid deficiency), diet, and lifestyle (reduction in calcium intake, lack of vitamin D, sedentary lifestyles) (Hauge et al., 2003; Iacono, 2007). Both Type I and Type II osteoporosis share the common effect of increased susceptibility to catastrophic fracture in bone.

Bone fragility due to osteoporosis has been examined in terms of changes in bone structure and resultant influence on mechanical properties. The ability of bone to resist catastrophic fracture depends on structure including bone mass, spatial distribution such as shape and micro-architecture, as well as the intrinsic properties of the bone material (Bouxsein, 2001). Bone fragility is therefore determined by the complex interaction between these parameters and, ultimately, the failure of mechanical function due to the diseased osteoporotic bone state (Turner, 2002). However, a range of bone mechanical properties are known to control catastrophic failure, particularly the strength (ultimate stress), stiffness (elastic modulus), and energy absorption quality (workto-fracture) of bone structures (Turner, 2002). Structural changes in osteoporotic bone at the macrostructural, architectural, and microstructural levels are typically diagnosed as a reduction of bone density. This density loss is consistent across the variety of organizations found in bone such that cortical bone displays a reduction in bone mass and trabecular bone exhibits thinning and loss of the number of trabecular struts across the body (Carter and Hayes, 1976; Wu et al., 2008; Kennedy et al., 2009; Zebaze et al., 2010) from the onset of osteoporosis. The stiffness and strength of trabecular bone are typically related to bone density in a non-linear fashion with either a squared (Rice et al., 1988), cubic (Carter and Hayes, 1976) or more complex (Marcus and Bouxsein, 2010) relationship to the change in density. However, this nonlinear relationship between bone density and resultant mechanical properties for trabecular bone has been explained by considering variations in bone volume fraction, trabecular orientation, trabecular interconnectivity, and structural anisotropy, which result in a linear relationship between bone density and mechanical properties for loading along the main trabecular orientation (Silva and Gibson, 1997; Keaveny et al., 2001). The geometry and organizational structure of osteoporotic bone therefore clearly define mechanical properties. Changes in material composition have 
been additionally identified as lowering strength in osteoporotic bone, particularly due to decreases in the degree of mineralization as porosity increases (Currey, 1988; Schaffler and Burr, 1988) or increases in mineralization due to the continuous aggregations of mineral without resorption (Grynpas, 1993). Additional compositional changes in cortical bone induced by osteoporosis include collagen content and orientation of collagen fibrils, the extent and nature of collagen cross-linking (Burr, 2002), as well as the number and composition of cement lines (Burr et al., 1988) that cause fatigue-induced micro-damage (Burr et al., 1997; Burr, 2003). Many of these latter factors affecting osteoporotic bone mechanical properties are more closely related to compositional changes at the smaller length scales existing at lower hierarchical levels. The structural changes in osteoporotic bone at higher hierarchical levels provide mechanical performance that is thus dependent on both the constituent material properties and the changes in bone geometry due to reduction in bone mass. However, the effect of osteoporosis on bone mechanics remains uncertain such that density alone cannot, for example, account for the decrease in stiffness and strength of trabecular bone, with strain to failure almost independent of density (Keaveny et al., 2001). The material properties of bone dominate at the lower hierarchical levels of bone where geometric and structural factors can be ignored. Thus, evaluating the quality of the bone material requires suitable mechanical tests at relatively small length scales.

The effect of the quality of osteoporotic bone on mechanical properties is important as current diagnosis methods purely based on bone density scales from x-ray scans are not optimal. For example, the National Osteoporosis Guideline Group (NOGG) has placed guidelines for the diagnosis of osteoporosis, which take into account the patient's medical history along with the $\mathrm{x}$-ray measured bone mineral density (BMD) index. BMD alone has been a poor indicator for potential increases in bone fragility and is only able to predict $60 \%$ of the variations in bone strength (Ammann and Rizzoli, 2003). The quality of the bone material brought on by a patient's lifestyles and other factors affecting the quality of bone material has been suggested as being an important consideration in determining bone fragility due to osteoporosis (WHO, 2012). Further techniques have been developed in order to quantify the mineral content of bone and assess the quality of bone material. These techniques include microradiography (Boivin and Baud, 1984; Boivin and Meunier, 2002), quantitative backscattered electron imaging (qBEI) (Roschger et al., 2003) and synchrotron radiation micro computed tomography (SR $\mu \mathrm{CT}$ ) (Borah et al., 2005). All of these methods perform measurements in what is referred to as bone mineralization density distribution (BMDD). BMDD is a measure of the mineral content in small areas defined as image pixels or voxels and can distinguish local variations in mineral content. BMD is a potentially poorer description of osteoporosis as an estimate of the total amount of mineral in a scanned area of whole bone, but is the current method used clinically (Roschger et al., 2008). Imaging techniques used to quantify bone mineral distribution have been previously combined with addition structural or mechanical testing, notably nanoindentation (Guo and Goldstein, 2000), scanning acoustic microscopy (SAM) (Katz and Meunier, 1993), Raman spectroscopy (McCreadie et al., 2006), and Fourier transform infrared imaging (FTIR) (Paschalis et al., 2004) in order to correlate mineral content to structure and function relationships (Roschger et al., 2008).

Compositional changes in bone material due to osteoporosis have been shown to decrease the degree of mineralization and collagen cross-linking, resulting in bone fragility (Paschalis et al., 2004; Marcus and Bouxsein, 2010). Reductions in the degree of mineralization have been further emphasized as detrimental to the material properties of bone (Ciarelli et al., 2003). The stiffness versus toughness of bone is determined in part by the mineral content (Currey, 1988; McCreadie et al., 2006) and exhibits significant degradation in mechanical properties with relatively small mineral content changes, which increase bone fragility (Roschger et al., 2008). In the case of osteoporosis, a decrease or an increase in mineralization may therefore be detrimental to the mechanical properties of bone (Ciarelli et al., 2003; Roschger et al., 2008). Low mineralization levels, or hypomineralization, cause reductions in stiffness and strength while high mineralization levels, or hypermineralization, reduce fracture toughness (Ciarelli et al., 2003). Hypomineralization occurs either due to lack of time for secondary mineralization to occur after bone remodeling or due to pathological conditions affecting mineralization. Conversely, hypermineralization only occurs when changes in crystal size or shape provide increased packing for a higher mineral density (Roschger et al., 2008). The significance of changes in the properties of bone material has led to works that attempt to measure mechanical properties of bone at small length scales, thus ignoring geometric effects at higher hierarchical levels. Notable experiments at the microstructural level were performed applying nanoindentation on trabecule from the lumbar region of 17-month-old control and ovariectomized (OVH) Sprague Dawley rats. These results showed no change in elastic modulus or hardness at the microscopic level between control and diseased specimens (Guo and Goldstein, 2000), suggesting that osteoporosis does not change the material properties of bone but instead only induces changes in bone density. A similar study by Maïmoun et al. (2012) showed a reduction in bone density due to a depletion of oestrogen in Sprague Dawley OVH rats, but a reduction in the elastic modulus in trabecular bone, which contradicted previous results. Additional studies attempting to assess the effect of osteoporosis on the mechanical properties of bone have also shown significant mechanical variations. Nanoindentation applied to cross-sections of osteoporotic and healthy bone of female human femurs was shown to give no change in elastic modulus even though the results of the qBEI analysis showed a lower mineralization level for the osteoporotic samples (FratzlZelman et al., 2009). This lack of a decrease in elastic modulus with lower mineralization in osteoporotic bone was attributed to changes in the organic matrix determining mechanical performance. Specifically, increasing the stiffness of the collagen fibrils of the organic matrix can occur with an increase in the crosslinking between protein chains, which may compensate for the low mineral content or a change in the mineral-organic interface during osteoporosis. Such an observation is important as consideration of changes in the softer organic phase in addition to variations in the volume fraction of the harder mineral must be considered in osteoporotic bone. The lack of clarity in osteoporotic bone mechanics is emphasized when considering aged bone, with 
nanoindentation showing an increase in the elastic modulus of osteoporotic bone (Silva et al., 2004) or decreasing strength, stated as due to decreasing mineral content and size distribution but increasing average crystal size (Boskey, 2003). The resultant variability in nanoindentation data makes correlation with structural and biochemical observations difficult. The testing environment may also contribute to listed variation in mechanical performance for osteoporotic, and indeed healthy, bone with a number of studies evaluating bone in a dehydrated state (Guo and Goldstein, 2000; Silva et al., 2004; Fratzl-Zelman et al., 2009). The diversity of literature evaluations of osteoporotic bone is summarized in Table 1 and includes comparative data on healthy bone. This table highlights the range of loading conditions used to determine the mechanical properties of bone. General comparisons between healthy and osteoporotic bone suggest small losses in strength and elastic modulus due to osteoporosis, with smaller length scale measurements providing higher absolute values than larger length scale measurements. The current paper therefore attempts to address the conflict in defining the effects of osteoporosis on bone by mechanically testing the material properties of bone. Techniques to isolate specific constituents and discrete volumes of bone have been previously shown to be effective in characterizing the material behavior (Hang and Barber, 2011; Hang et al., 2011, 2014; JimenezPalomar et al., 2012). Of these studies, the ability to mechanically test discrete volumes of bone is particularly beneficial for understanding the synergy between constituents while removing the effects of sample geometry (Jimenez-Palomar et al., 2012). Microbeams selected using focused ion beam (FIB) microscopy have been previously employed to understand the mechanical properties of biological materials including teeth (Chan et al., 2009) and bone (Jimenez-Palomar et al., 2012). This work therefore exploits micro-beams from cortical bone but expands on the technique to evaluate the effects of osteoporosis on resultant bone material mechanics.

\section{MATERIALS AND METHODS}

The bone of rats is commonly used as a model for osteoporosis, which can be induced through estrogen depravation by performing an ovariectomy (Frost and Jee, 1992; Guo and Goldstein, 2000). $\mathrm{OVH}$ and control rat femurs were obtained from the Hebrew University of Jerusalem with ethical approval in order to compare the mechanical properties of osteoporotic and healthy bone. The diaphysis from the extracted rat femur was first isolated using a water-cooled diamond blade slow speed circular saw (Buehler, USA) to produce a bone sample with approximate dimensions of $12 \mathrm{~mm} \times 1 \mathrm{~mm} \times 1 \mathrm{~mm}$. The bone sample was stored in $70 \%$ ethanol: $30 \%$ water solution overnight followed by submerging within progressively increasing ethanol solutions of 85,95 , and $100 \%$ for 60,30 , and $120 \mathrm{~min}$, respectively, to provide sample dehydration. The dehydrated sample was then transferred to the chamber of a small dual beam system (SDB, Quanta 3D, FEI Company, EU/USA) for subsequent FIB milling. Micro-beams of bone were created using FIB as detailed in Jimenez-Palomar et al. (2012) using conditions to remove FIB damage in soft materials as described previously (Bailey et al., 2013). The FIB preparation method can be summarized in a series of steps where bone material was first removed rapidly, followed by more precision FIB removal at smaller length scales to produce micro-beams with regular geometries. The corner of the macroscopic bone sample was first cleaned to produce orthogonal surfaces at the sample edge using a high current ion beam of $65 \mathrm{nA}$ and accelerating voltage of $30 \mathrm{kV}$. Flattening of the bone surfaces was achieved by further FIB removal of smaller bone volumes using smaller ion beam currents down to $0.1 \mathrm{nA}$. These smaller ion beam currents

Table 1 | Mechanical properties of osteoporotic and comparative healthy rat bone taken from the literature.

\begin{tabular}{|c|c|c|c|c|c|}
\hline & State & Testing method & Strength (MPa) & Elastic modulus (GPa) & Reference \\
\hline \multicolumn{6}{|c|}{ MACROSTRUCTURAL LEVEL } \\
\hline Whole bone & Healthy & 3-Point bending & $180 \pm 6$ & $6.9 \pm 0.3$ & Jorgensen et al. (1991) \\
\hline Whole bone & Healthy & 3-Point bending & $134 \pm 4$ & $8 \pm 0.4$ & Barengolts et al. (1993) \\
\hline Whole bone & Healthy & 3-Point bending & $153 \pm 45$ & $4.9 \pm 4$ & Ejersted et al. (1993) \\
\hline \multicolumn{6}{|l|}{ ARCHITECTURAL LEVEL } \\
\hline Cross section ( $1 \mathrm{~mm}$ thick) & Healthy (cortical) & Compression & $139.5 \pm 19.14$ & $8.8 \pm 2.5$ & Cory et al. (2010) \\
\hline Cross section ( $1 \mathrm{~mm}$ thick) & Osteoporotic (cortical) & Compression & $127.24 \pm 35.04$ & $7.3 \pm 2.7$ & Cory et al. (2010) \\
\hline Cross section ( $1 \mathrm{~mm}$ thick) & Healthy (trabecular) & Compression & $35.95 \pm 15.62$ & $2.2 \pm 0.92$ & Cory et al. (2010) \\
\hline Cross section ( $1 \mathrm{~mm}$ thick) & Osteoporotic (trabecular) & Compression & $26.89 \pm 22.35$ & $1.02 \pm 0.79$ & Cory et al. (2010) \\
\hline Beams (1 mm thick) & Healthy & 3-Point bending & - & $5.12 \pm 0.77$ & Kasra et al. (1997) \\
\hline Beams (1 mm thick) & Osteoporotic & 3-Point bending & - & $4.70 \pm 0.98$ & Kasra et al. (1997) \\
\hline \multicolumn{6}{|c|}{ SUB-MICROSTRUCTURAL LEVEL (SUB-LAMELLAR/MATERIAL LEVEL) } \\
\hline Lamellar (25 $\mu \mathrm{m}^{2}$ indent) & Healthy (trabecular) & Nanoindentation & - & $16.1 \pm 3.9$ & Guo and Goldstein (2000) \\
\hline Lamellar (25 $\mu \mathrm{m}^{2}$ indent) & Osteoporotic (trabecular) & Nanoindentation & - & $15.8 \pm 3.9$ & Guo and Goldstein (2000) \\
\hline Lamellar & Healthy (cortical) & Nanoindentation & - & $18.98 \pm 4.78$ & Cory et al. (2010) \\
\hline Lamellar & Healthy (trabecular) & Nanoindentation & - & $18.27 \pm 4.26$ & Cory et al. (2010) \\
\hline Lamellar & Healthy (trabecular) & Nanoindentation & - & $18.73 \pm 0.71$ & Maïmoun et al. (2012) \\
\hline Lamellar & Osteoporotic (trabecular) & Nanoindentation & - & $16 \pm 0.85$ & Maïmoun et al. (2012) \\
\hline Lamellar & Healthy (cortical) & Nanoindentation & - & $21.27 \pm 1.2$ & Maïmoun et al. (2012) \\
\hline Lamellar & Osteoporotic (cortical) & Nanoindentation & - & $21.12 \pm 1.12$ & Maïmoun et al. (2012) \\
\hline
\end{tabular}


avoid observable ion beam damage. FIB milling was additionally performed parallel to the produced sample faces in all preparation steps to reduce embedding the impinging gallium ions from the FIB within the discrete beam volumes produced. A short column between each of the micro-beams was retained in order to prevent the re-deposition of milled material and gallium ions on to neighboring beams. Thus, bone material sputtered from the FIB is more likely to redeposit on the short columns instead of the sample micro-beams. The average micro-beam dimensions produced at the end of the bone material sample were $8 \mu \mathrm{m} \times 2 \mu \mathrm{m} \times 2 \mu \mathrm{m}$, with the long axis of the micro-beam aligned in the direction of the long axis of the femur. These dimensions at micron length scales are comparable to bone lamellae and therefore remove structural features present at larger length scales.

Resultant bone micro-beams were removed from the SDB setup and placed in a closed vessel containing Hank's buffer solution for $2 \mathrm{~h}$ to allow bone rehydration. Samples were then returned to the SDB system for subsequent mechanical testing, with the prior sample rehydration shown to preserve the mechanical properties of the wet bone for up to $2 \mathrm{~h}$ in such an environment (Jimenez-Palomar et al., 2012). Mechanical testing of the bone micro-beams was carried out using an atomic force microscope (AFM) integrated within the SDB (Hang et al., 2011). A physiologically relevant compressive loading configuration was used, with the load applied in the direction of the long axis of the micro-beams. The AFM allowed the application of load to the micro-beams while scanning electron microscopy (SEM) within the SDB system was used to observe the deformation and resultant failure of the samples. Mechanical testing was achieved by first translating the AFM tip toward individual bone micro-beams until compressive load was applied parallel to the micro-beam long axis as shown in Figure 1. In situ SEM was used to observe the movement of the AFM tip toward the end of the micro-beam and ensure that the AFM tip fully contacted the top of the beam as shown in Figure 2. Compression of four osteoporotic and six healthy bone micro-beams was carried out under quasi-static loading rates.

\section{RESULTS}

Compression of bone micro-beams using the AFM produced corresponding data for the force applied to the sample and resultant deformation. Figure 2 shows SEM imaging highlighting the loading of the micro-beam sample in compression until failure of the sample occurred. The stress and strain induced in the bone microbeams were calculated using the force-deflection curves generated from the AFM system. Stress is calculated by dividing the force applied to the sample from the AFM tip by the cross-sectional area of the micro-beam sample, measured from SEM, whereas strain is calculated by dividing the change in length by the total micro-beam sample length, as shown in Eqs 1 and 2 below.

$$
\begin{aligned}
\sigma & =\frac{f}{A} \\
\varepsilon & =\frac{\Delta L}{L_{0}}
\end{aligned}
$$

Where $\sigma$ is the stress in the compressed micro-beam sample, $f$ is the force applied by the AFM, $A$ is the micro-beam cross-sectional

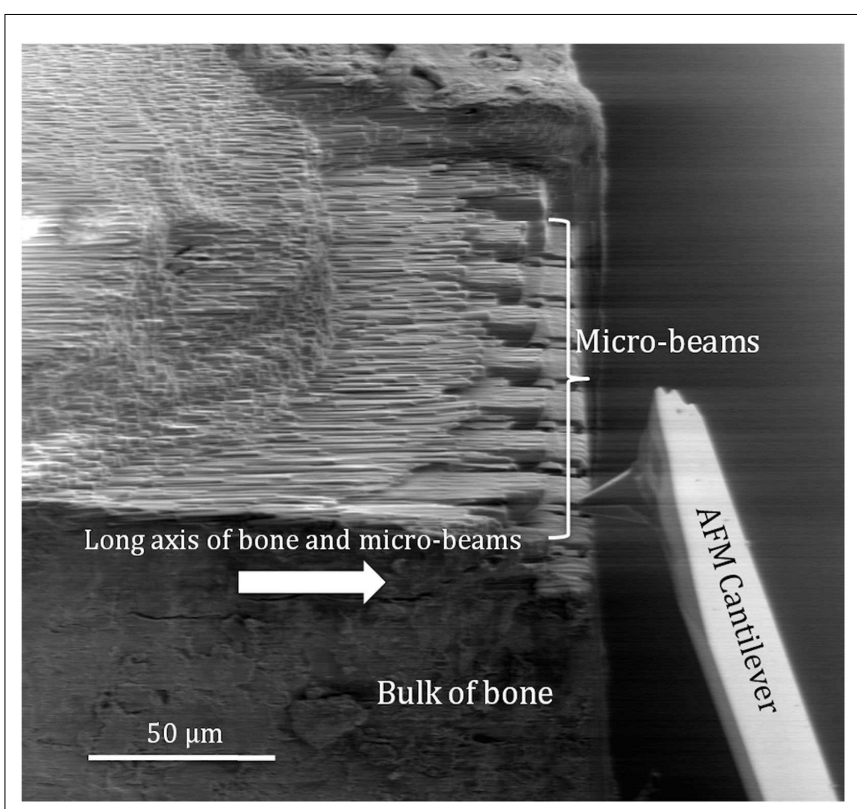

FIGURE 1 | Scanning electron micrograph of the AFM tip attached to the force detection system of the AFM cantilever approaching a bone micro-beam for in situ mechanical testing. The arrow indicates the direction of the long axis of bone and micro-beam principal axis.

area, $\varepsilon$ is the strain in the bone micro-beam, $\Delta L$ is change in length of the beam, and $L_{0}$ is the original length of the bone microbeam prior to mechanical deformation. The resultant stress-strain behavior for the osteoporotic and control bone micro-beams is shown in Figure 3. The stress generally increases in a relatively linear manner with increasing strain until failure of the microbeam. We note that local non-linearity is due to the interferometer measurement system of the AFM as described previously (Hang et al., 2011). The elastic modulus, strength, and the strain to failure values calculated from the bone micro-beam compression tests in Figure 3 are shown in Table 2 for the healthy bone control and the osteoporotic OVH model samples. The OVH bone has an average elastic modulus of $1.59 \pm 1.26 \mathrm{GPa}$, almost half the value of the elastic modulus of $2.9 \pm 1.45 \mathrm{GPa}$ for the control sample. The OVH and control samples exhibit similar strength values of $169.23 \pm 21.35$ and $169.51 \pm 66.19 \mathrm{MPa}$, respectively. A larger average strain to failure of $10 \%$ is recorded for the OVH samples when compared to $\sim 6 \%$ for control samples. The toughness of the bone defined by the area under the stress-strain curves is $\sim 8$ and $5 \mathrm{~J} \cdot \mathrm{m}^{-3}$ for $\mathrm{OVH}$ and control samples, respectively. The increased toughness displayed for the OVH samples is surprising as osteoporotic bone is commonly associated with brittle failure. However, we reiterate that the work presented here examines the material properties of bone and the enhanced toughness is due to increased strain to failure of the material. The fragility of osteoporotic bone associated with larger or whole bone samples is therefore absent when evaluating the small-scale material performance.

The mechanical property values recorded from the microbeam compression of this work generally lie within the architectural range of previous literature as listed in Table 1 . The 

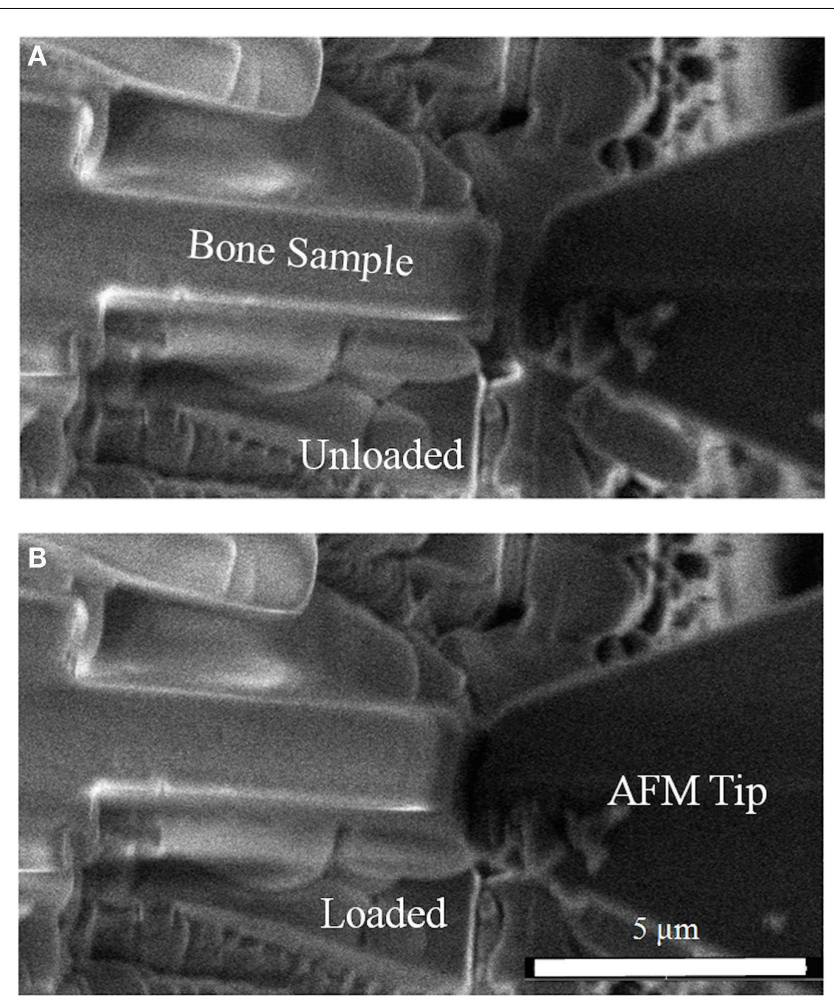

FIGURE 2 | Scanning electron micrograph showing compression of rat bone micro-beams (A) in the unloaded state with the AFM tip away from the bone micro-beam and (B) during contact of the AFM tip with the bone micro-beam causing compressive loading.

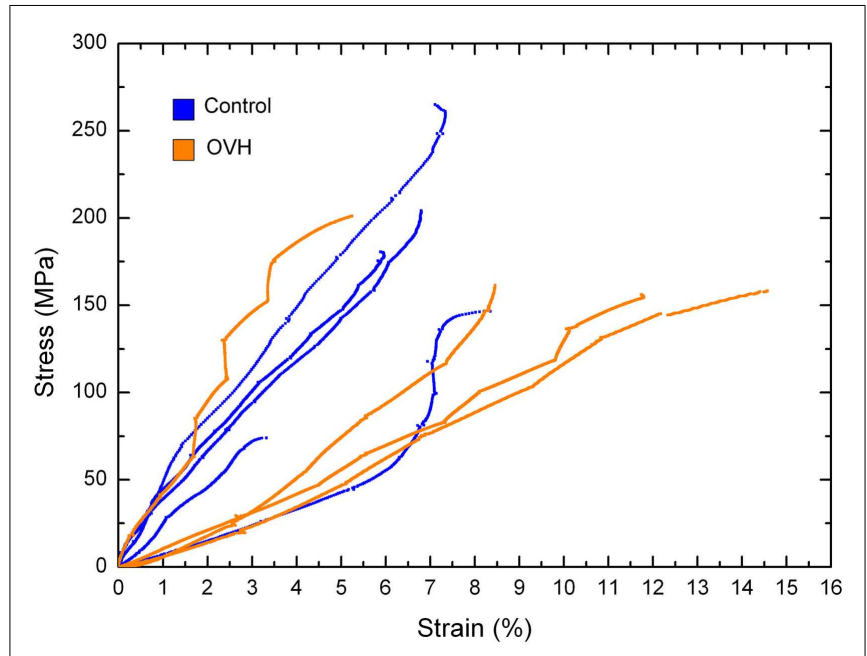

FIGURE 3 | Stress-strain curves for compression of control and ovariectomized (OVH) rat bone micro-beams.

average elastic modulus values in our micro-beam compression show larger variations between osteoporotic and healthy bone samples than the results in Table 1, highlighting the sensitivity of the technique in elucidating mechanical changes in bone
Table 2 | Elastic modulus, strength, and strain to failure values of both control and ovariectomized (OVH) rat femur bone micro-beams tested in compression.

\begin{tabular}{cccc}
\hline Beam no. & Elastic modulus (GPa) & Strength (MPa) & Strain (\%) \\
\hline Control & & & \\
Average & $2.9 \pm 1.45$ & $169.51 \pm 66.19$ & $6.3 \pm 1.89$ \\
1 & 3.06 & 204.22 & 6.8 \\
4 & 3.62 & 180.53 & 5.88 \\
5 & 2.37 & 73.91 & 3.32 \\
6 & 4.65 & 248.35 & 7.16 \\
8 & 0.78 & 140.52 & 8.35 \\
OVH & & & \\
Average & $1.59 \pm 1.26$ & $169.23 \pm 21.35$ & $10 \pm 4.04$ \\
1 & 3.46 & 201.09 & 5.24 \\
2 & 1.08 & 156.17 & 8.46 \\
3 & 1.07 & 161.38 & 11.74 \\
4 & 0.74 & 158.26 & 14.57 \\
\hline
\end{tabular}

material due to osteoporosis. Indeed, error associated with the average elastic modulus values is expected to be due to variability in the orientation of collagen fibrils within the micro-beam that is measurable using the experimental setup. We suggest that this enhanced mechanical sensitivity of the technique is due to the removal of sample geometry effects, such as shape of the bone or porosity that is found at larger length scales, using FIB. Many bone structures also have strain to failure values considerably lower than our values and this may be attributed to the lack of geometric effects, such as porosity, which provide strain concentrations locally whereas the bulk of the material remains at lower strain. Indeed, glassy polymers such as polystyrene are analogous to this potential situation where mechanical testing of smaller volumes of material removes the effect of defects, causing increases in strain to failure (van der Sanden et al., 1993). Other mechanical testing techniques such as nanoindentation typically probe significantly smaller volumes than the micro-beams of this work and are potentially sensitive to more variability from the location of the indenting probe at the sample surface, with considerable issues related to the uncertainty in the composition of the material and resultant contact area with the indenting probe previously reviewed (Lewis and Nyman, 2008). The enhanced sensitivity of the micro-beam compression in our work indicates a clear decrease of bone elastic modulus properties with osteoporosis as shown in Figure 3, which potentially contradicts some works in Table 1 that indicate little variability in the elastic modulus of osteoporotic bone compared to healthy bone. This lowering of the elastic modulus of osteoporotic bone has been suggested as being due to mechanical degradation of the collagen in osteoporotic bone from reductions in the level of immature collagen crosslinks and decreases in collagen fibril diameters (Currey, 2003). Compositional changes in collagen, such as the ratio of $\alpha 1$ to $\alpha 2$ chains in different phenotypes of COLIA1 found in Type 1 collagen, appear to influence the fracture risk of bone that is independent to the changes in bone mass (McGuigan et al., 2001). A corresponding decrease in bone strength is not observed in our 
work and suggests that the failure of the material does not change with osteoporosis although the interactions between constituents are affected. Indeed, molecular modeling has indicated considerable variation in mechanical behavior of collagen fibrils as a function of cross-linking density, including significant changes in elastic modulus as well as regime of cross-linking densities that provide minimal changes in strength (Buehler, 2008). We would therefore expect a decrease in elastic modulus of OVH samples as the stress transfer between protein molecules becomes inefficient from the previously reported changes in cross-linking density. Such a mechanism can additionally describe the lack of a loss in strength as the same collagen protein molecules are failing in both the healthy and OVH samples. The proposed mechanism here therefore describes deformation and failure of osteoporotic bone in terms of protein from the collagen in bone. Non-collagenous proteins (NCPs) present between collagen fibrils have also been shown to control fracture behavior of bone (Hang et al., 2014) and are known to chemically change in osteoporotic bone (Sroga and Vashishth, 2012). While the performance of osteoporotic bone material has been defined in this paper, the origin of the mechanical changes is still contentious, with collagen, mineral, NCPs, and their interactions all potentially contributing to mechanics. Such a complex synergy has been highlighted previously when considering compensation mechanisms where increased stiffness for the organic phase is balanced by a decrease in mineral content that results in similar nanoindentation hardness for diseased and healthy bone (Fratzl-Zelman et al., 2009). Future development of mechanistic explanations for osteoporotic bone therefore requires a comprehensive understanding of all constituent materials together.

\section{CONCLUSION}

The compressive elastic modulus, strength, and strain to failure of bone micro-beams were measured in order to assess the effect of osteoporosis on the mechanical properties of bone as a material at the sub-lamellar level. Although compression testing herein cannot be directly compared to previous studies in the literature, results showed a decrease in the elastic modulus of osteoporotic bone compared to a control. This decrease in the elastic modulus with osteoporosis was additionally associated with relatively constant micro-beam strength and a small increase in failure strain, with associated changes in material toughness. The origin of osteoporotic induced decreases in bone elastic modulus was suggested as being due to mechanical degradation of the collagen within the bone material.

\section{REFERENCES}

Ammann, P., and Rizzoli, R. (2003). Bone strength and its determinants. Osteoporos. Int. 14, 13-18. doi:10.1007/s00198-002-1345-4

Bailey, R. J., Geurts, R., Stokes, D. J., Jong, F. D., and Barber, A. H. (2013). Evaluating focused ion beam induced damage in soft materials. Micron 50, 51-56. doi:10.1016/j.micron.2013.04.005

Barengolts, E. I., Curry, D. J., Bapna, M. S., and Kukreja, S. C. (1993). Effects of endurance exercise on bone mass and mechanical properties in intact and ovariectomized rats. J. Bone Miner. Res. 8, 937-942. doi:10.1002/jbmr. 5650080806

Boivin, G., and Baud, C. A. (1984). "Microradiographic methods for calcified tissues," in Methods for Calcified Tissue Preparation, ed. G. R. Dickson (Amsterdam: Elsevier), 391-411.
Boivin, G., and Meunier, P. J. (2002). The degree of mineralization of bone tissue measured by computerized quantitative contact microradiography. Calcif. Tissue Int. 70, 503-511. doi:10.1007/s00223-001-2048-0

Borah, B., Ritman, E. L., Dufresne, T. E., Jorgensen, S. M., Liu, S., Sacha, J., et al. (2005). The effect of risedronate on bone mineralization as measured by microcomputed tomography with synchrotron radiation: correlation to histomorphometric indices of turnover. Bone 37, 1-9. doi:10.1016/j.bone.2005.03.017

Boskey, A. (2003). Bone mineral crystal size. Osteoporos. Int. 14, 16-21. doi:10.1007/ s00198-003-1468-2

Bouxsein, M. (2001). "Biomechanics of age-related fractures," in Osteoporosis, 2nd Edn, eds R. Marcus, D. Feldman, and J. Kelsey (San Diego, CA: Academic Press), 509-534.

Buehler, M. J. (2008). Nanomechanics of collagen fibrils under varying cross-link densities: atomistic and continuum studies. J. Mech. Behav. Biomed. Mater. 1, 59-67. doi:10.1016/j.jmbbm.2007.04.001.

Burr, D. (2003). Microdamage and bone strength. Osteoporos. Int. 14, S67-S72. doi:10.1007/s00198-003-1476-2

Burr, D. B. (2002). The contribution of the organic matrix to bone's material properties. Bone 31, 8-11. doi:10.1016/S8756-3282(02)00815-3

Burr, D. B., Forwood, M. R., Fyhrie, D. P., Martin, R. B., Schaffler, M. B., and Turner, C. H. (1997). Bone microdamage and skeletal fragility in osteoporotic and stress fractures. J. Bone Miner. Res. 12, 6-15. doi:10.1359/jbmr.1997.12.1.6

Burr, D. B., Schaffler, M. B., and Frederickson, R. G. (1988). Composition of the cement line and its possible mechanical role as a local interface in human compact bone. J. Biomech. 21, 939-945. doi:10.1016/0021-9290(88)90132-7

Carter, D. R., and Hayes, W. C. (1976). Bone compressive strength: the influence of density and strain rate. Science 149, 1174-1176. doi:10.1126/science.996549

Chan, Y. L., Ngan, A. H. W., and King, N. M. (2009). Use of focused ion beam milling for investigating the mechanical properties of biological tissues: a study of human primary molars. J. Mech. Behav. Biomed. Mater. 2, 375-383. doi:10.1016/j.jmbbm.2009.01.006

Ciarelli, T. E., Fyhrie, D. P., and Parfitt, A. M. (2003). Effects of vertebral bone fragility and bone formation rate on the mineralization levels of cancellous bone from white females. Bone 32, 311-315. doi:10.1016/S8756-3282(02)00975-4

Cory, E., Nazarian, A., Entezari, V., Vartanians, V., Müller, R., and Snyder, B. D. (2010). Compressive axial mechanical properties of rat bone as functions of bone volume fraction, apparent density and micro-ct based mineral density. J. Biomech. 43, 953-960. doi:10.1016/j.jbiomech.2009.10.047

Currey, J. D. (1988). The effect of porosity and mineral content on the young's modulus of elasticity of compact bone. J. Biomech. 21, 131-139. doi:10.1016/00219290(88)90006-1

Currey, J. D. (2003). Role of collagen and other organics in the mechanical properties of bone. Osteoporos. Int. 14, S29-S36. doi:10.1007/s00198-003-1470-8

Ejersted, C., Andreassen, T. T., Oxlund, H., Jorgensen, P. H., Bak, B., Haggblad, J., et al. (1993). Human parathyroid hormone (1-34) and (1-84) increase the mechanical strength and thickness of cortical bone in rats. J. Bone Miner. Res. 8 , 1097-1101. doi:10.1002/jbmr.5650080910

Fratzl-Zelman, N., Roschger, P., Gourrier, A., Weber, M., Misof, B. M., Loveridge, N., et al. (2009). Combination of nanoindentation and quantitative backscattered electron imaging revealed altered bone material properties associated with femoral neck fragility. Calcif. Tissue Int. 85, 335-343. doi:10.1007/s00223-0099289-8

Frost, H. M., and Jee, W. S. S. (1992). On the rat model of human osteopenias and osteoporoses. Bone Miner. 18, 227-236. doi:10.1016/0169-6009(92)90809-R

Grynpas, M. (1993). Age and disease-related changes in the mineral of bone. Calcif. Tissue Int. 53, S57-S64. doi:10.1007/BF01673403

Guo, X. E., and Goldstein, S. A. (2000). Vertebral trabecular bone microscopic tissue elastic modulus and hardness do not change in ovariectomized rats. J. Orthop. Res. 18, 333-336. doi:10.1002/jor.1100180224

Hang, F., and Barber, A. H. (2011). Nano-mechanical properties of individual mineralized collagen fibrils from bone tissue. J. R. Soc. Interface 8, 500-505. doi:10.1098/rsif.2010.0413

Hang, F., Gupta, H. S., and Barber, A. H. (2014). Nanointerfacial strength between non-collagenous protein and collagen fibrils in antler bone. J. R. Soc. Interface 11, 20130993. doi:10.1098/rsif.2013.0993

Hang, F., Lu, D., Bailey, R. J., Jimenez-Palomar, I., Stachewicz, U., Cortes-Ballesteros, B., et al. (2011). In situ tensile testing of nanofibers by combining atomic force microscopy and scanning electron microscopy. Nanotechnology 22, 365708. doi:10.1088/0957-4484/22/36/365708 
Hauge, E. M., Steiniche, T., and Andreassen, T. T. (2003). "Histomorphometry of metabolic bone conditions," in Handbook of Histology Methods for Bone and Cartilage, eds Y. H. An and K. L. Martin (Totowa, NJ: Human Press Inc), 391-410.

Iacono, M. V. (2007). Osteoporosis: a national public health priority. J. Perianesth. Nurs. 22, 175-183. doi:10.1016/j.jopan.2007.03.009

Jimenez-Palomar, I., Shipov, A., Shahar, R., and Barber, A. H. (2012). Influence of SEM vacuum on bone micromechanics using in situ AFM. J. Mech. Behav. Biomed. Mater. 5, 149-155. doi:10.1016/j.jmbbm.2011.08.018

Jorgensen, P. H., Bak, B., and Andreassen, T. T. (1991). Mechanical properties and biochemical composition of rat cortical femur and tibia after longterm treatment with biosynthetic human growth hormone. Bone 12, 353-359. doi:10.1016/8756-3282(91)90022-B

Kasra, M., Vanin, C. M., Maclusky, N. J., Casper, R. F., and Grynpas, M. D. (1997). Effects of different estrogen and progestin regimens on the mechanical properties of rat femur. J. Orthop. Res. 15, 118-123. doi:10.1002/jor.1100150117

Katz, J. L., and Meunier, A. (1993). Scanning acoustic microscope studies of the elastic properties of osteons and osteon lamellae. J. Biomech. Eng. 115, 543-548. doi:10.1115/1.2895537

Keaveny, T. M., Morgan, E. F., Niebur, G. L., and Yeh, O. C. (2001). Biomechanics of trabecular bone. Annu. Rev. Biomed. Eng. 3, 307-333. doi:10.1146/annurev. bioeng.3.1.307

Kennedy, O. D., Brennan, O., Rackard, S. M., Staines, A., O’Brien, F. J., Taylor, D., et al. (2009). Effects of ovariectomy on bone turnover, porosity, and biomechanical properties in ovine compact bone 12 months postsurgery. J. Orthop. Res. 27, 303-309. doi:10.1002/jor.20750

Kilbanski, A., Adams-Campbell, L., Bassford, T., Blair, S. N., Boden, S. D., Dickersin, K., et al. (2001). Osteoporosis prevention, diagnosis, and therapy. JAMA 285, 785-795. doi:10.1001/jama.285.6.785

Lewis, G., and Nyman, J. S. (2008). The use of nanoindentation for characterizing the properties of mineralized hard tissues: state-of-the art review. J. Biomed. Mater. Res. B Appl. Biomater. 87, 286-301. doi:10.1002/jbm.b.31092

Maïmoun, L., Brennan-Speranza, T. C., Rizzoli, R., and Ammann, P. (2012). Effects of ovariectomy on the changes in microarchitecture and material level properties in response to hind leg disuse in female rats. Bone 51, 586-591. doi:10.1016/j.bone.2012.05.001

Marcus, R., and Bouxsein, M. L. (2010). “The nature of osteoporosis," in Fundamentals of Osteoporosis, eds R. Marcus, D. Feldman, D. A. Nelson, and C. J. Rosen (San Diego, CA: Academic Press), 25-34.

McCreadie, B. R., Morris, M. D., Chen, T., Sudhaker, R. D., Finney, W. F., Widjaja, E., et al. (2006). Bone tissue compositional differences in women with and without osteoporotic fracture. Bone 39, 1190-1195. doi:10.1016/j.bone.2006.06.008

McGuigan, F. E. A., Armbrecht, G., Smith, R., Felsenberg, D., Reid, D. M., and Ralston, S. H. (2001). Prediction of osteoporotic fractures by bone densitometry and COLIA1 genotyping: a prospective, population-based study in men and women. Osteoporos. Int. 12, 91-96. doi:10.1007/s001980170139

Paschalis, E. P., Shane, E., Lyritis, G., Skarantavos, G., Mendelsohn, R., and Boskey, A. L. (2004). Bone fragility and collagen cross-links. J. Bone Miner. Res. 19, 2000-2004. doi:10.1359/jbmr.040820

Rice, J. C., Cowin, S. C., and Bowman, J. A. (1988). On the dependence of the elasticity and strength of cancellous bone on apparent density. J. Biomech. 21, 155-168. doi:10.1016/0021-9290(88)90008-5
Roschger, P., Gupta, H. S., Berzlanovich, A., Ittner, G., Dempster, D. W., Fratzl, P., et al. (2003). Constant mineralization density distribution in cancellous human bone. Bone 32, 316-323. doi:10.1016/S8756-3282(02)00973-0

Roschger, P., Paschalis, E. P., Fratzl, P., and Klaushofer, K. (2008). Bone mineralization density distribution in health and disease. Bone 42, 456-466. doi:10.1016/j. bone.2007.10.021

Schaffler, M. B., and Burr, D. B. (1988). Stiffness of compact bone: effects of porosity and density. J. Biomech. 21, 13-16. doi:10.1016/0021-9290(88)90186-8

Silva, M. J., Brodt, M. D., Fan, Z., and Rho, J. Y. (2004). Nanoindentation and wholebone bending estimates of material properties in bones from the senescence accelerated mouse SAMP6. J. Biomech. 37, 1639-1646. doi:10.1016/j.jbiomech. 2004.02.018

Silva, M. J., and Gibson, L. J. (1997). Modeling the mechanical behavior of vertebral trabecular bone: effects of age-related changes in microstructure. Bone 21, 191-199. doi:10.1016/S8756-3282(97)00100-2

Sroga, G. E., and Vashishth, D. (2012). Effects of bone matrix proteins on fracture and fragility in osteoporosis. Curr. Osteoporos. Rep. 10, 141-150. doi:10.1007/ s11914-012-0103-6

Turner, C. H. (2002). Biomechanics of bone: determinants of skeletal fragility and bone quality. Osteoporos. Int. 13, 97-104. doi:10.1007/s001980200000

van der Sanden, M. C. M., Meijer, H. E. H., and Lemstra, P. J. (1993). Deformation and toughness of polymeric systems: 1 . The concept of critical thickness. Polymer 34, 2148-2154. doi:10.1016/0032-3861(93)90249-A

WHO. (2012). National Osteoporosis Guideline Group (NOGG) [Online]. Sheffield: University of Sheffield. Available at: http://www.shef.ac.uk/NOGG/

Wu, Z.-X., Lei, W., Hu, Y.-Y., Wang, H.-Q., Wan, S.-Y., Ma, Z.-S., et al. (2008). Effect of ovariectomy on BMD, micro-architecture and biomechanics of cortical and cancellous bones in a sheep model. Med. Eng. Phys. 30, 1112-1118. doi:10.1016/j.medengphy.2008.01.007

Zebaze, R. M. D., Ghasem-Zadeh, A., Bohte, A., Iuliano-Burns, S., Mirams, M., Price, R. I., et al. (2010). Intracortical remodelling and porosity in the distal radius and post-mortem femurs of women: a cross-sectional study. Lancet 375, 1729-1736. doi:10.1016/S0140-6736(10)60320-0

Conflict of Interest Statement: The authors declare that the research was conducted in the absence of any commercial or financial relationships that could be construed as a potential conflict of interest.

Received: 12 December 2014; paper pending published: 29 December 2014; accepted: 23 January 2015; published online: 13 February 2015.

Citation: Jimenez-Palomar I, Shipov A, Shahar R and Barber AH (2015) Mechanical behavior of osteoporotic bone at sub-lamellar length scales. Front. Mater. 2:9. doi: 10.3389/fmats.2015.00009

This article was submitted to Mechanics of Materials, a section of the journal Frontiers in Materials.

Copyright (C) 2015 Jimenez-Palomar, Shipov, Shahar and Barber. This is an openaccess article distributed under the terms of the Creative Commons Attribution License (CC BY). The use, distribution or reproduction in other forums is permitted, provided the original author(s) or licensor are credited and that the original publication in this journal is cited, in accordance with accepted academic practice. No use, distribution or reproduction is permitted which does not comply with these terms. 